\title{
Improvements in medium range weather forecasting system of India
}

\author{
V S Prasad*, Saji Mohandas, Surya Kanti Dutta, M Das Gupta, \\ G R Iyengar, E N Rajagopal and Swati Basu \\ ${ }^{1}$ National Centre for Medium Range Weather Forecasting, A-50, Sector 62, Noida 201 309, India. \\ ${ }^{*}$ Corresponding author.e-mail: prasadvs@gmail.com
}

Medium range weather forecasts are being generated in real time using Global Data Assimilation Forecasting System (GDAFS) at NCMRWF since 1994. The system has been continuously upgraded in terms of data usage, assimilation and forecasting system. Recently this system was upgraded to a horizontal resolution of T574 (about $22 \mathrm{~km}$ ) with 64 levels in vertical. The assimilation scheme of this upgraded system is based on the latest Grid Statistical Interpolation (GSI) scheme and it has the provision to use most of available meteorological and oceanographic satellite datasets besides conventional meteorological observations. The new system has an improved procedure for relocating tropical cyclone to its observed position with the correct intensity. All these modifications have resulted in improvement of skill of medium range forecasts by about 1 day.

\section{Introduction}

The operational global data assimilation and forecast system (GDAFS) of NCMRWF (an adapted version of NCEP GFS system) has undergone various changes in terms of data usage, model physics, resolution and analysis schemes, etc. since 1989 (table 1). The changes were made with an aim to increase forecast skill by incorporating latest advances in model physics, analysis schemes and skill to incorporate new types of datasets into the model. The increase in computing resources at the centre over the years was also an important factor in implementing the abovementioned changes (table 2). A schematic diagram of the entire operational NWP system at NCMRWF is given in figure 1. The meteorological observations from all over the globe and from various observing platforms are received at Regional Telecommunication Hub (RTH), New Delhi through Global
Telecommunication System (GTS) and the same is made available to NCMRWF through a dedicated link. Special arrangements have been made to receive bulk satellite data directly from satellite operators, viz., NOAA-NESDIS, EUMETSAT and ISRO (via ftp access). All data are decoded from their native format and encoded into NCEP Binary Universal Form for the representation of meteorological data (BUFR) format using various decoders and are archived in a special file structure, called BUFR-TANK, for subsequent use. The global data assimilation system (GDAS) accesses the observational database from the BUFR-TANK at a specific time each day (every 6 hours, starting from 0000 hours), four times a day.

The latest major changes that were implemented in May 2011 were significant not only in terms of the improved model physics, but also in the capability of decoding and assimilating more conventional and satellite observations. Figure 1(b)

Keywords. Medium range forecast; data assimilation; numerical weather prediction. 
Table 1. History of global data assimilation and forecasting systems of NCMRWF.

\begin{tabular}{lccc}
\hline Model & $\begin{array}{c}\text { Analysis } \\
\text { scheme }\end{array}$ & Year & $\begin{array}{c}\text { Forecasting } \\
\text { range (days) }\end{array}$ \\
\hline R40L18 & OI & 1989 & 5 \\
T80L18 & SSI & 1992 & 5 \\
T170L28 & SSI & 2005 & 5 \\
T170L42 & SSI & 2006 & 5 \\
T254L64 & SSI & 2007 & 7 \\
T254L64 & GSI & 2008 & 7 \\
T382L64 & GSI & 2010 & 10 \\
T574L64 & GSI & 2010 & 10 \\
\hline
\end{tabular}

Table 2. History of high performance computing (HPC) at NCMRWF.

\begin{tabular}{lll}
\hline Sl. no & HPC (with peak performance) & Period \\
\hline 1 & Cray XMP-14 (4 MFlops) & $1988-1993$ \\
2 & Cray XMP-216(16 MFlops) & $1993-1998$ \\
3 & Dec-Alpha cluster (9.6 GFlops) & $1999-2011$ \\
4 & Cray SV1 (28.8 GFlops) & $2001-2006$ \\
5 & PARAM Padma (500 GFlops) & $2006-2011$ \\
6 & Cray X1E (1.1 TFlops) & $2006-2011$ \\
7 & IBM Power 6 (24 TFlops) & 2010 onwards \\
\hline
\end{tabular}

shows increase in the volume of data, in both via GTS and ftp, from time to time. The horizontal resolution of the new GDAFS is now T574 (about $22 \mathrm{~km}$ ) but vertical resolution is kept same at 64 levels similar to previous version (T382L64). The 64 unequally spaced hybrid sigma-pressure levels are terrain following near the lower levels and constant pressure surfaces in the stratosphere and beyond. There are 15 levels below $800 \mathrm{hPa}$ and 24 levels above $200 \mathrm{hPa}$. The implementation details of this new GDAFS as well as the preliminary results are given in Prasad et al. (2011). The performance of two variants of NCMRWF global analysis forecast systems with resolutions T382L64 and T574L64 in predicting the various components of the Indian summer monsoon during 2011 were evaluated. The salient features of the latest global data assimilation-forecast systems vis-à-vis the previous version and the significant results from the same are also presented in this paper.

\section{Forecast model}

Rajagopal et al. (2007) give a detailed description of the NCMRWF GFS system which was operational from January 2007 at NCMRWF. The forecast model is a primitive equation global spectral model in hybrid sigma-pressure vertical coordinates (Moorthi et al. 2001; Environmental
Modeling Centre 2003) for vorticity, divergence, $\ln (\mathrm{Ps})$, virtual temperature and three tracers (specific humidity, ozone mixing ratio and cloud condensate mixing ratio). The model horizontal resolutions are T382 and T574 with $1152 \times 576$ $(\sim 35 \mathrm{~km})$ and $1760 \times 880$ Gaussian grid points $(\sim 22 \mathrm{~km})$, respectively near the equatorial latitudes and employ a reduced Gaussian grid (64 and 18 grid points respectively) as it goes towards either poles.

Time integration is leapfrog for nonlinear advection terms. Semi-implicit method is used for gravity waves and for zonal advection of vorticity and specific humidity. A time split physics adjustment scheme is employed with implicit treatment when possible and damping to simulate subgrid scale dissipation. A digital filter initialization with a $3 \mathrm{~h}$ window is used for mass and wind balance and (Asselin 1972) time filter is used to reduce computational modes. The model's time step for T382L64 and T574L64 are 3 and 2 min, respectively. Mean orographic heights on the Gaussian grid are used. Horizontal diffusion is 8th order scale selective for divergence, vorticity, virtual temperature (on quasi-pressure surfaces), specific humidity, ozone and cloud-condensate. Vertical advection of tracers caused computationally generated negative tracers, which are minimized by a positivedefinite tracer transport scheme in T574L64 implementation. This change was necessary for the newly implemented GSI which is sensitive to the negative water vapour. 

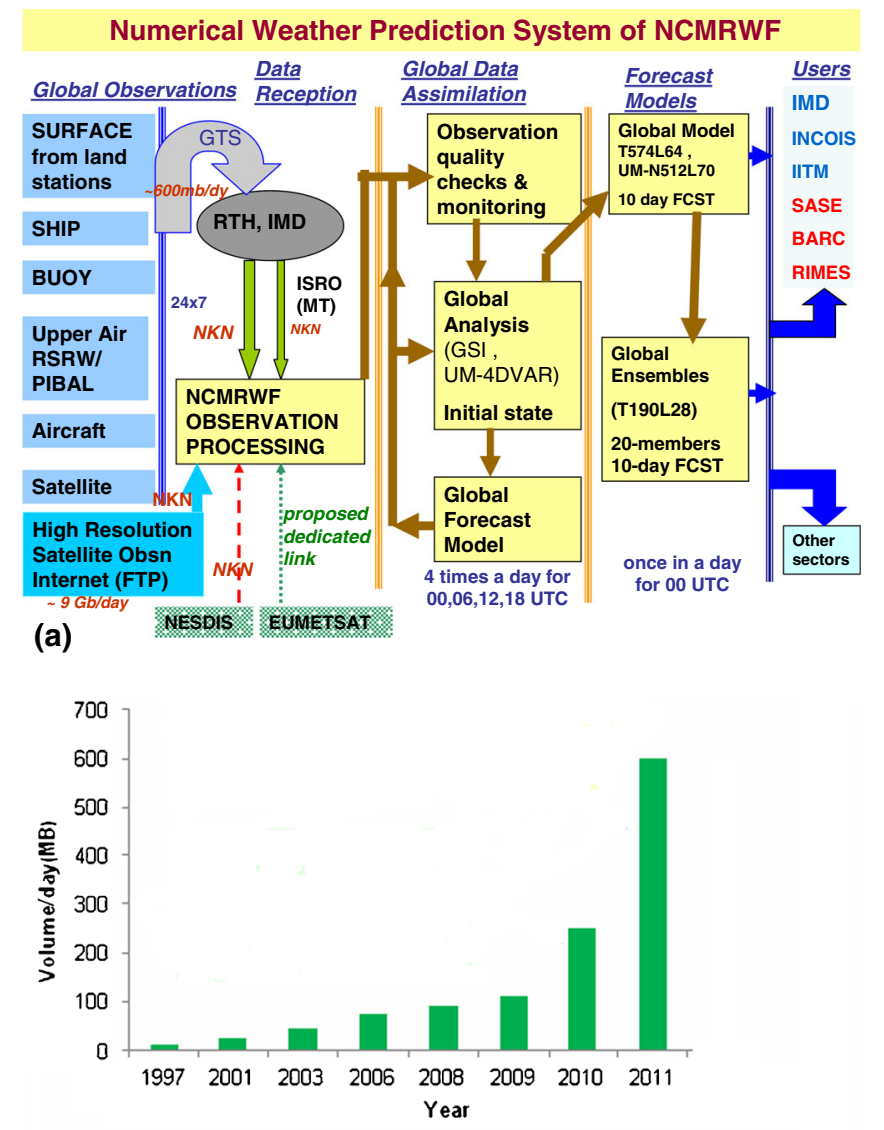

Volume of data reception through GTS (MB/day)

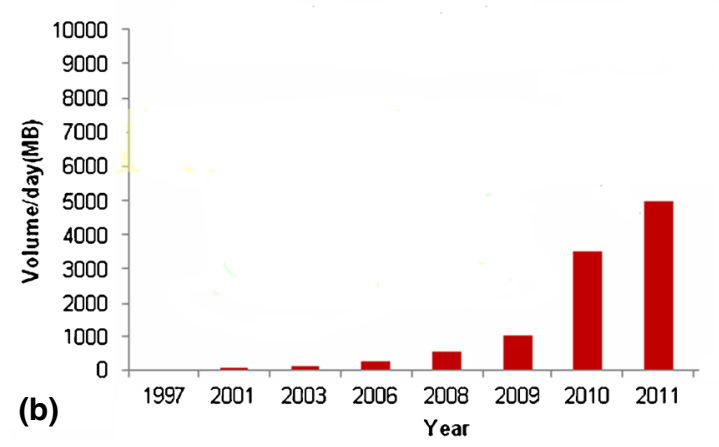

Volume of data reception through FTP via internet(MB/day

Figure 1. (a) Schematic diagram of the entire operational NWP system at NCMRWF. (b) Showing year-to-year increase of data received at and used at NCMRWF.

The major changes incorporated in the present GDAFS implementation compared to the previous T382L64 are tabulated in table 3. New T574L64 is an upgraded version of NCEP GFS system (Kanamitsu 1989; Kalnay et al. 1990; Kanamitsu et al. 1991; Moorthi et al. 2001; EMC 2003). The physics changes are mainly in radiation, gravity wave drag, planetary boundary layer processes, shallow and deep convection schemes and an introduction of tracer transport scheme in the vertical (Saha et al. 2010). Short wave (SW) routine is changed from RRTM to RRTM1 and invoked every hour. Long wave (LW) computation frequency is $1 \mathrm{~h}$. Stratospheric aerosols (for both SW and LW) and tropospheric aerosols (LW) are added. In stratosphere, for shortwave computation the aerosol single scattering albedo is changed from 0.90 to 0.99 . SW aerosol asymmetry factor is changed and the new aerosol climatology (Sato et al. 1993) is used. SW cloud overlap is changed from random to maximum random overlap. Time varying global mean $\mathrm{CO}_{2}$ is used instead of constant $\mathrm{CO}_{2}$. Treatment of the dependence of direct-beam surface albedo on solar zenith angle over snow-free land surface is based on Yang et al. (2008).

Gravity wave drag (GWD) has also been modified to automatically scale mountain block and GWD stress with resolution (Kim and Arakawa 1995; Lott and Miller 1997), using four times stronger mountain block and one half the strength of GWD. Planetary boundary layer (PBL) processes include stratocumulus-top driven turbulence mixing, enhanced stratocumulus-top driven diffusion for cloud top entrainment instability and local diffusion for night time stable PBL. Background diffusion in inversion layer of $2.5 \mathrm{~km}$ over ocean is reduced by $70 \%$ to decrease the erosion of stratocumulus along the coastal area. Bulk-Richardson number is used to calculate PBL height.

Deep convection parameterization follows modified Simplified-Arakawa Scheme (SAS) (Han and Pan 2010). It eliminates random cloud type, and detrain cloud water from every cloud layer of the height cloud. Finite entrainment and detrainment rates for heat, moisture and momentum are specified. Similar to shallow convection scheme, entrainment rate is given to be inversely proportional to height in subcloud layers and detrainment rate is set to be a constant as entrainment rate at the cloud base. Above cloud base, an organized entrainment is added, which is a function of environmental relative humidity. Intraseasonal momentum background diffusivity is applied for winds only. Convective overshooting increased cloud water detrainment in upper cloud layers.

There are many causes for negative water vapour generation in NWP models, the biggest contributor being the usage of vertical central differencing scheme. Removal of this major cause of negative water vapour was affected by a positivedefinite tracer transport scheme (Yang et al. 2009) in the vertical to replace the central-differencing scheme to eliminate computationally-induced negative tracers. This included the change in parameters 'factqmin and factqmax' in GSI, to reduce negative water vapour and super-satauration points from analysis steps. Modifying cloud physics to limit the borrowing of water vapour that is 


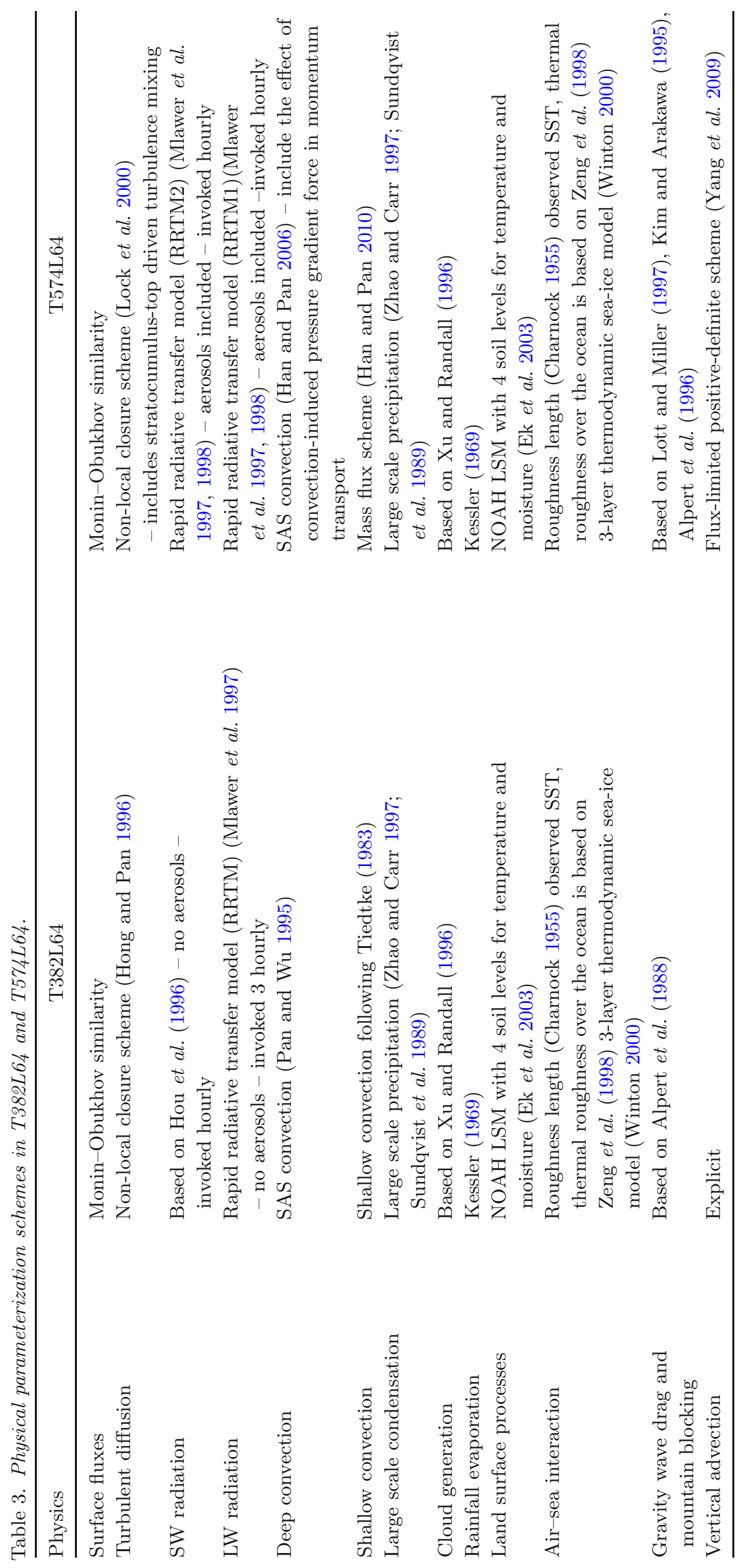


used to fill negative cloud water to the maximum amount of available water vapour so as to prevent the model from producing negative water vapour, the minimum value of water vapour mass mixing ratio in the radiation is changed from $1.0 \mathrm{e}-5$ to $1.0 \mathrm{e}-20$. Otherwise the model artificially injects water vapour in the upper atmosphere where water vapour mixing ratio is often below $1.0 \mathrm{e}-5$.

At NCMRWF, vertical coordinate was changed from sigma to hybrid sigma pressure, reducing some upper air model errors (Sela 2009) in this latest change. The hybrid coordinate system is terrain following in the lower levels and transforming to pure pressure coordinate in the upper levels and is uniquely represented by two values $A_{k}$ and $B_{k}$ at the interfaces. The values of $\left(A_{k} * 1000.0\right)$ and $B_{k}$ are read from an input file. The interface pressure levels (in $\mathrm{hPa}$ ) can be derived by the relation;

$$
\text { IPres }_{k+\frac{1}{2}}=\left(A_{k+\frac{1}{2}}+B_{k+\frac{1}{2}} \cdot P_{0}\right) 10.0,
$$

where $P_{0}$ is the surface pressure in centi-bars (cb).

The layer pressures (Pres), sigma interface (SI) and sigma layer (SL) equivalents for dynamics can be obtained by mathematical manipulations.

\section{Analysis scheme}

The global analysis scheme used at NCMRWF earlier was spectral statistical interpolation (SSI). However from January 2008, SSI system was replaced with grid-point statistical interpolation (GSI). The key aspect of the GSI is that it formulates the analysis in model grid space, which allows for more flexibility in the application of the background error co-variances and makes it straightforward for a single analysis system to be used across a broad range of applications, including both global and regional modelling systems. In the GSI system many new features are included, such as changes to the observation selection, quality control, minimization algorithm, dynamic balance constraint and assimilation of new observation types.

GSI scheme (Wu et al. 2002) is an evolutionary combination of the SSI scheme and the regional ETA 3D-VAR. It replaces spectral definition for background errors with grid point (physical space) version based on recursive filters. This global 3DVAR in physical space is as effective as 3DVAR in spectral space with latitude-dependent structure functions and other error statistics. Diagonal background error covariance in spectral space (in SSI) allows little control over the spatial variation of the error statistics as the structure function is limited to being geographically homogeneous and isotropic about its center (Parrish and Derber 1992; Courtier et al. 1998). GSI allows greater flexibility in terms of inhomogeneity and anisotropy for background error statistics (Wu et al. 2002). Thus major improvement of GSI over SSI analysis scheme is its latitude-dependent structure functions and has more appropriate background errors in the tropics. The background error covariances are isotropic and homogeneous in the zonal direction. Thus results from initial

Table 4. Differences in the GSI scheme used in T574L64 and T382L64 systems.

\begin{tabular}{lc}
\hline New observations assimilated & Improvements in data assimilation system \\
\hline Inclusion of METOP IASI (Infrared Atmospheric & Use of variational qc \\
Sounding Interferometer) data & \\
Reduction of number of AIRS (Atmospheric Infrared & Addition of background error covariance input file \\
Sounder) water vapour channels used & \\
Assimilating tropical storm pseudo sea-level pressure & Flow dependent reweighting of background \\
observations & error variances \\
NOAA-19 HIRS/4 (High Resolution Infrared & Use of new version and coefficients for \\
Radiation Sounder) and AMSU-a (Advanced & community radiative transfer model (CRTM-2.02) \\
Microwave Sounding Unit) brightness temperature & \\
NOAA-18 SBUV/2 (Solar Backscatter Ultraviolet & Improved tropical cyclone relocation \\
Spectral Radiometer) Ozone, EUMETSAT-9 & \\
atmospheric motion vectors & Change in land/snow/ice skin temperature variance \\
Using uniform thinning mesh for brightness & \\
temperature data & \\
Improving assimilation of GPS radial occultation data & \\
RE-tuned observation errors & \\
ASCAT (Advanced Scatterometer) winds included & \\
Korean AMDAR data and more number of aircraft & \\
reports & \\
\hline
\end{tabular}


experiments reported that GSI had a small impact on extra-tropics but it had shown consistent positive impact in tropics (Wu et al. 2002). The full details of GSI scheme operational at NCMRWF can be found in Surya and Prasad (2011). Table 4 depicts the major differences in T382L64 and this new implementation.

\section{Results and discussions}

The two variants of NCMRWF global analysis forecast systems with resolutions T382L64 and T574L64 respectively were run in parallel for the entire monsoon season of 2011. A number of diagnostics have been generated to evaluate the performance of the GFS for the entire monsoon season of 2011.

The forecast errors (defined as the difference between the forecasts and the analyses) of the NCMRWF T574 GFS have been compared with those of the NCEP Operational GFS for JuneAugust (JJA) 2011. The diagnostics of the performance of the NCEP operational GFS have been obtained from NCEP (through personal communication). Figure 2(a and $\mathrm{b}$ ) shows the day-5 forecast errors of $850 \mathrm{hPa}$ zonal wind of the NCMRWF GFS and the NCEP GFS, respectively. It is seen that the forecast errors from the two systems are similar.

Further, objective verification scores for the both T382L64 and T574L64 forecasts were computed against the radiosonde observations valid at 00 UTC and also against their mean analyses at standard pressure levels as recommended by WMO (WMO-No. 485: Manual on the Global Dataprocessing and Forecasting System) for the entire monsoon season (June-September) of 2011. The verification scores are computed for analyses against observations, and for forecasts against both analyses and observations.

Figures 3 and 4 depict the vertical profile of T574L64 and T382L64 analyses and first guess vector wind fits to radiosonde observation for JJAS (June-September) over the global and global tropics $\left(30^{\circ} \mathrm{S}\right.$ to $\left.30^{\circ} \mathrm{N}\right)$ domains, respectively. The fits include bias and RMSE. The bold lines represent T382L64 and the dotted lines T574L64. The black and red lines represent the analyses and the first guess, respectively. The right panel in the plots gives the count of observation in thousands over the said region used for the comparison. Over the global domain (figure 3), a remarkable reduction in the RMSE values is seen in the T574L64 guess field as compared to the T382L64 guess field, in all levels and more pronounced in the upper levels. This indicates a tremendous improvement in the skill of the short range forecasts (first guess)

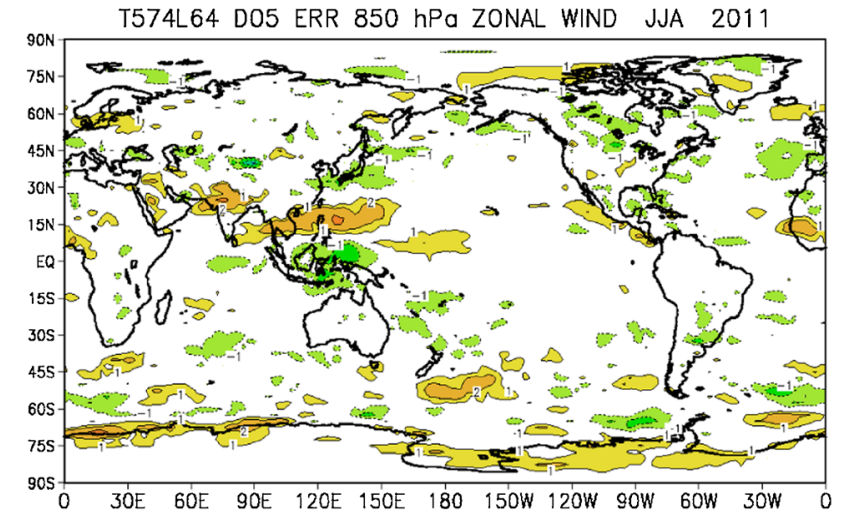

(a)

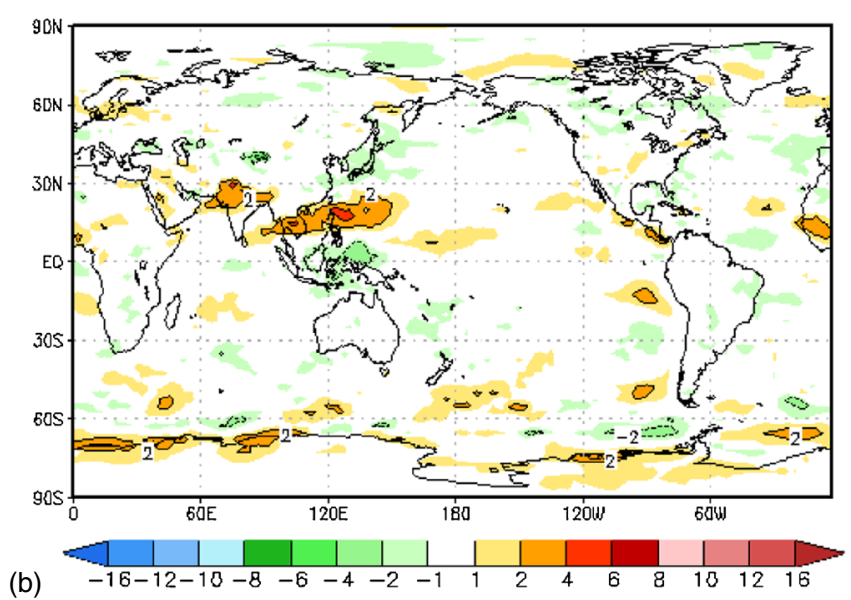

Figure 2. Day-5 $850 \mathrm{hPa}$ zonal wind forecast errors, w.r.t corresponding analysis for the period June-August 2011. (a) NCMRWF GFS and (b) NCEP GFS.

of the T574L64 model compared to the T382L64. The improvement is due to both changes in assimilation and model physics. The RMSE values in the T574L64 analysis field also show a marginal reduction as compared to the T382L64 analysis field. Similar results are seen over the tropics (figure 4) and other domains (figures not shown) to establish and confirm the improvement in the T574L64 analyses in comparison to the T382L64 analyses.

Figure 5 depicts the anomaly correlation for day-1 to day-10 forecasts of $500 \mathrm{hPa}$ geopotential height from the T574L64 and T382L64 models over the northern hemisphere. In the upper panel, the black and the red lines represent the T382L64 and T574L64 model, respectively. The T574L64 model shows higher values of anomaly correlation throughout the length of the 10 days forecast period. An anomaly correlation of 0.6 (Kalnay et al. 1998) is generally regarded as an indication of useful forecast. The T574L64 model is able to retain the value of anomaly correlation of 0.6 for one more day as compared to the T382L64 model, 


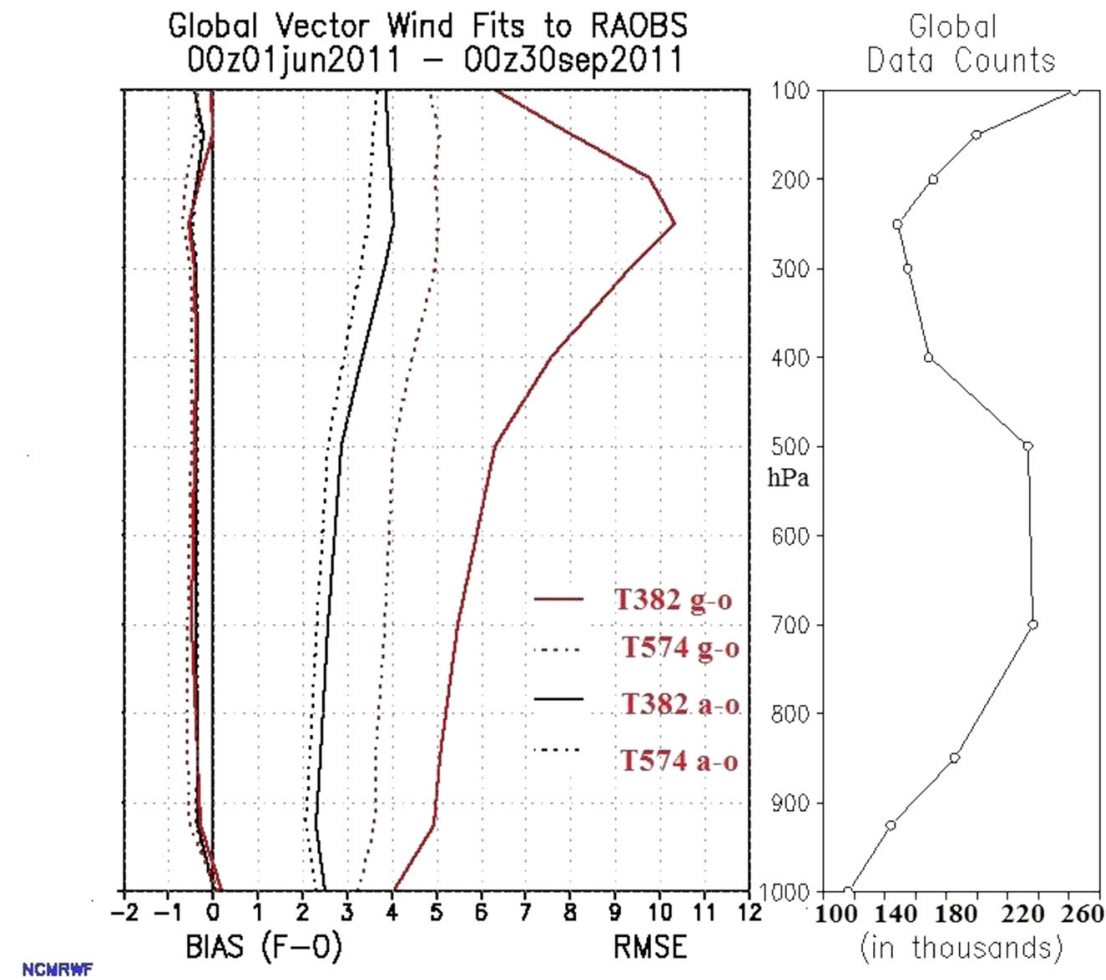

Figure 3. Global mean vector wind fits to radiosonde observations for 1 June 30-September 2011 at 00 UTC along with the observational data counts.

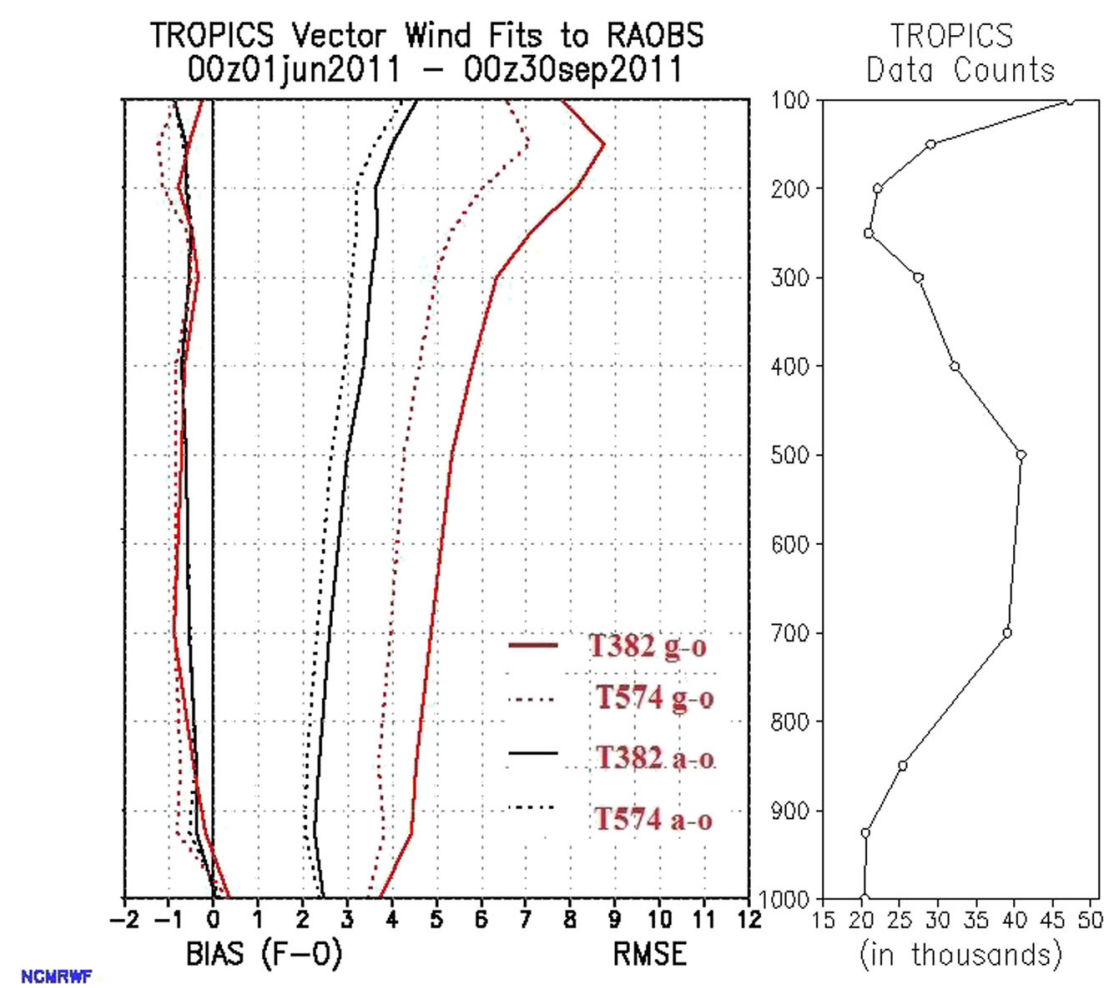

Figure 4. Same as figure 3, but for global tropics. 


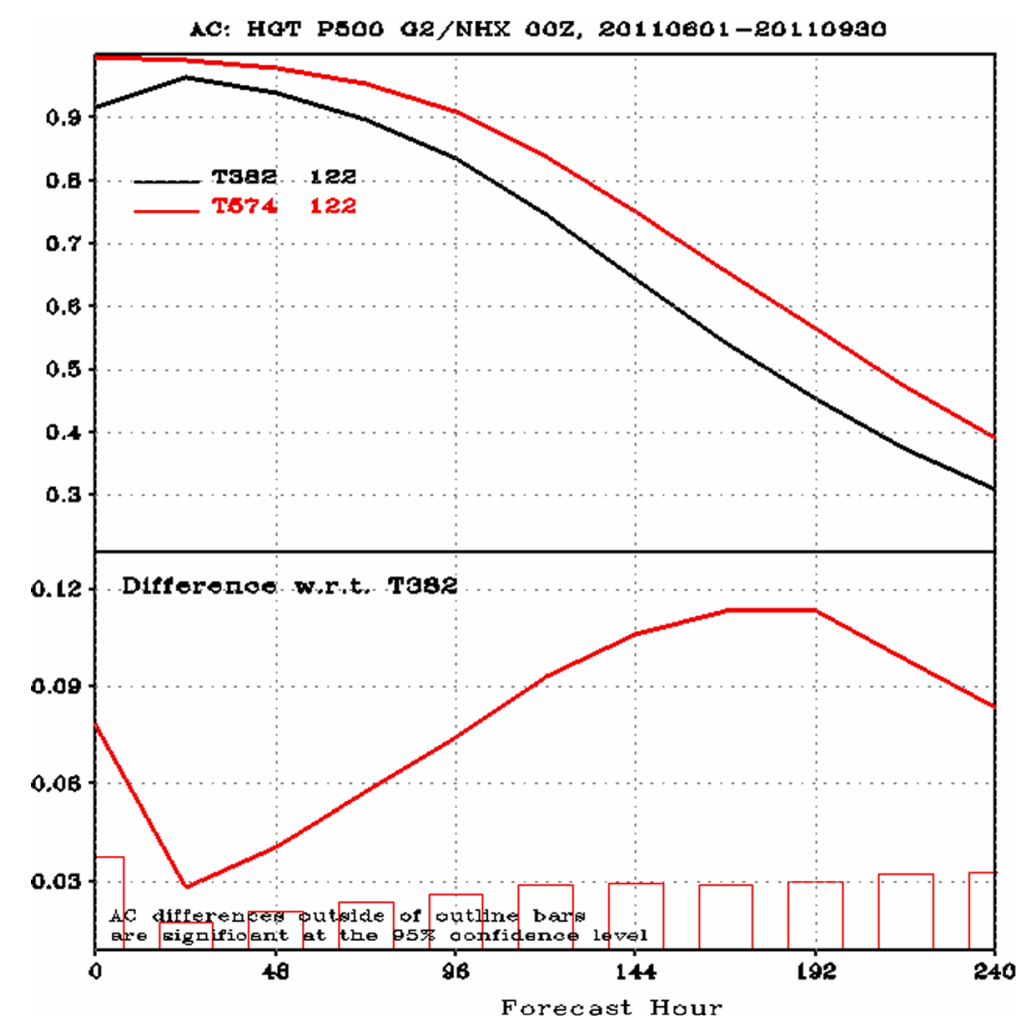

Figure 5. Anomaly correlation of $500 \mathrm{hPa}$ geopotential height for day-1 to day-10 forecasts over the northern hemisphere region (upper part of the figure) and anomaly correlation difference of T574L64 forecasts with respect to T382L64 forecasts along with their statistical significance (lower part of the figure).

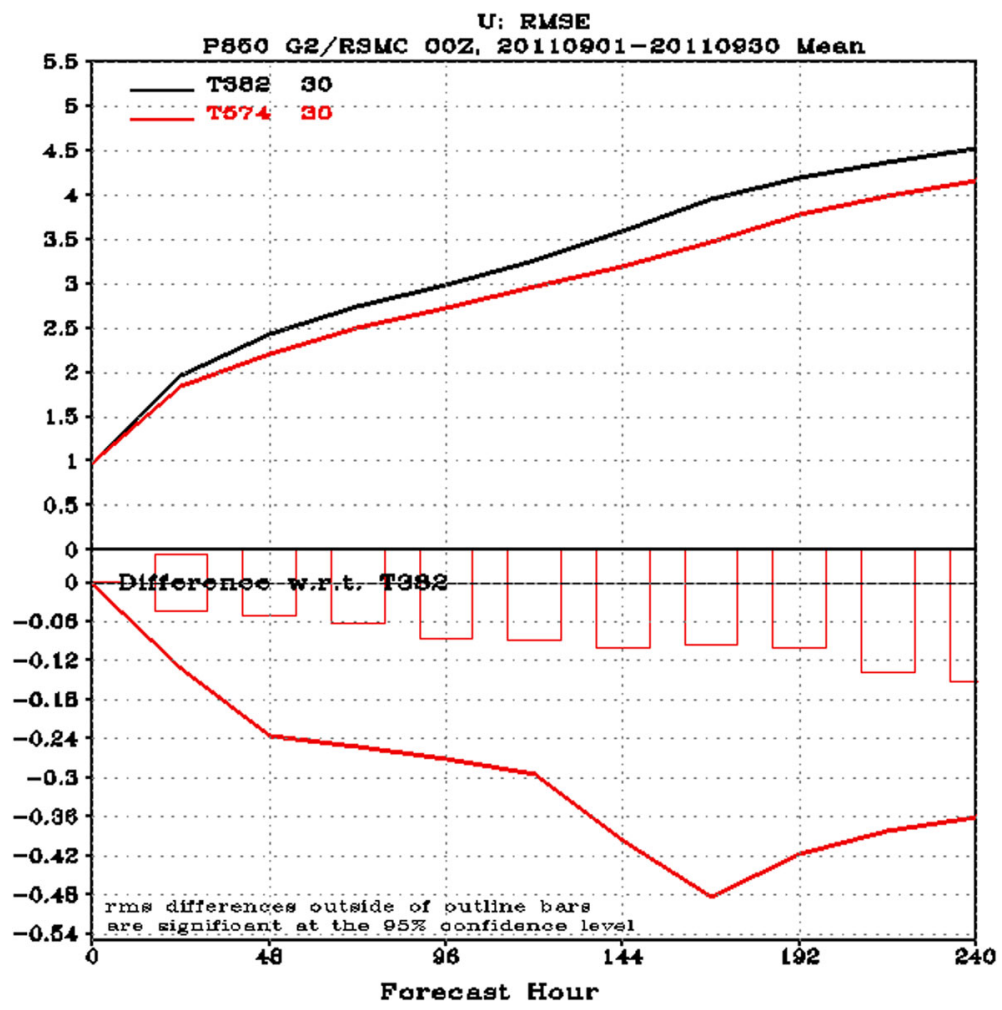

Figure 6. RMSE of $850 \mathrm{hPa}$ zonal wind over the RSMC region for day-1 to day-10 forecasts (upper part of the figure) and RMSE difference of T574L64 forecasts with respect to T382L64 forecasts along with their statistical significance (lower part of the figure). 


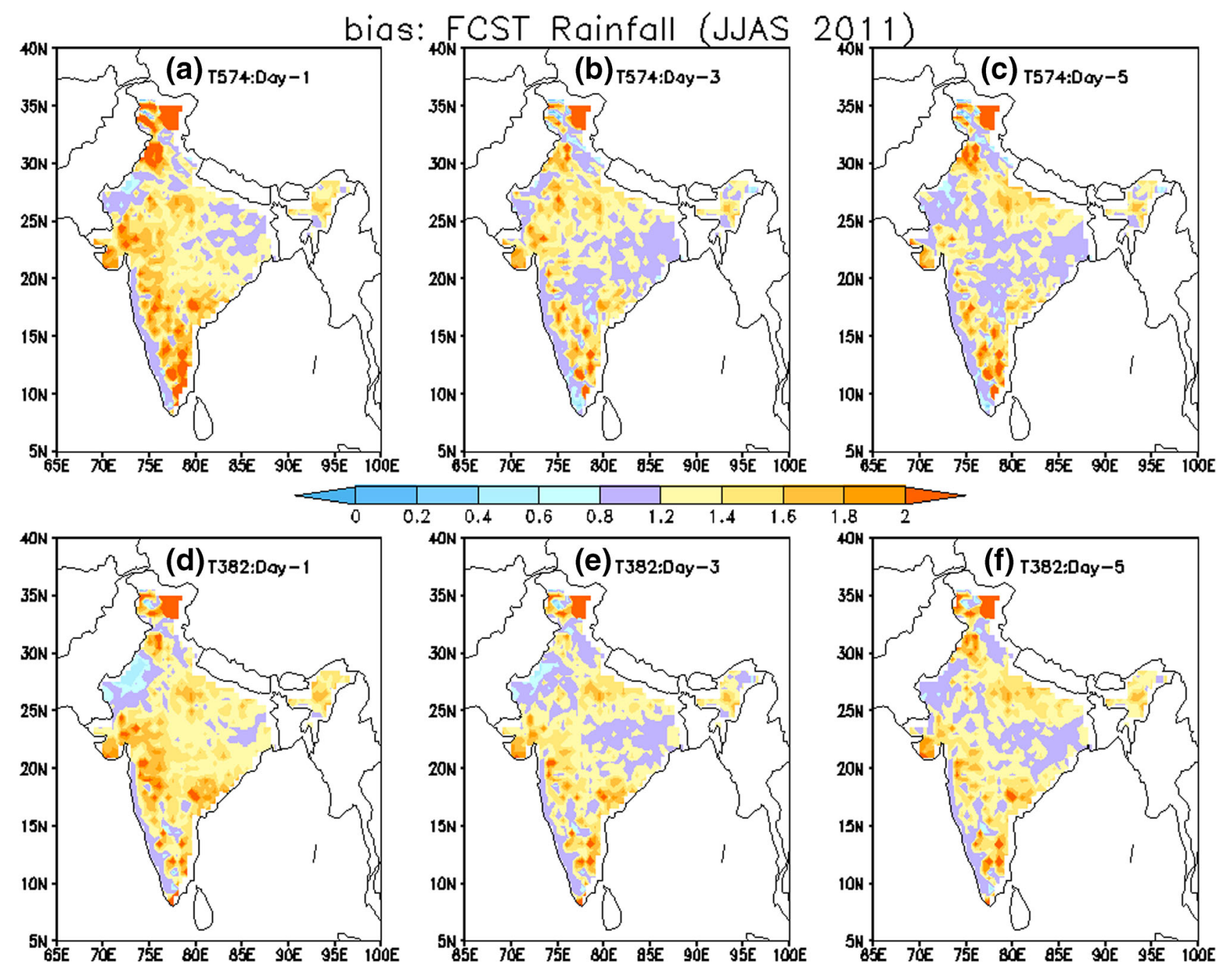

Figure 7. Frequency bias score of rainfall computed for day-1, day-3 and day-5 forecasts for the period June-September 2011 from T382L64 and T574L64 models.

thereby indicating an improvement of skill of the forecasts by about 1 day. In the lower panel, the line plot depicts the difference of the forecasts of $500 \mathrm{hPa}$ geopotential height of the T574L64 model from the T382L64 model. The difference values outside the histograms are statistically significant at $95 \%$ level of confidence determined by p-, as test. The T574L64 model is found to have significantly higher anomaly correlation at $95 \%$ level of confidence compared to T382L64 for all the days of the forecasts.

Figure 6 depicts the RMSE for day- 1 to day-10 forecasts of $850 \mathrm{hPa}$ zonal wind from the T574L64 and T382L64 models over the RSMC (Regional Specialized Meteorological Centre $\left(10^{\circ} \mathrm{S}-40^{\circ} \mathrm{N}\right.$, $\left.40^{\circ}-100^{\circ} \mathrm{E}\right)$ region of IMD. In the upper panel, the black and the red lines represent the T382L64 and T574L64 model, respectively. The T574L64 model shows lower values of RMSE throughout the length of the 10 days forecast period. In the lower panel, the line plot depicts the difference of the forecasts of $850 \mathrm{hPa}$ zonal wind of the T574L64 model from the T382L64 model. The difference values outside the histograms are statistically significant at $95 \%$ level of confidence. The T574L64 model is found to have significantly lower values of RMSE at $95 \%$ level of confidence compared to T382L64 for all the days of the forecasts.

\subsection{Indian summer monsoon rainfall}

The impact of the new system on the Indian summer monsoon is studied in terms of bias score. The bias score is a measure of the agreement between the forecast frequency and the observed frequency of 'yes' events. It is given by the ratio of the frequency of forecast events to the frequency of observed events. The bias score does not measure how well the forecast corresponds to the observations, it only measures relative frequencies. The score values range from 0 to infinity and the score of 1 implies a perfect forecast. It indicates whether 
Table 5. Contingency table illustrating the counts used in verification statistics for dichotomous (e.g., Yes/No) forecasts and observations.

\begin{tabular}{lll}
\hline & \multicolumn{2}{c}{ Observed } \\
\cline { 2 - 3 } Forecast & Yes & \multicolumn{1}{c}{ No } \\
\hline Yes & Hits & False alarms \\
No & Misses & Correct rejection \\
\hline
\end{tabular}

the forecast system has a tendency to underpredict $($ BIAS $<1)$ or overpredict $($ BIAS $>1)$ events.

$$
\text { BIAS }=\frac{\text { hits }+ \text { false alarms }}{\text { hits }+ \text { misses }}
$$

Figure 7 shows bias score computed for day-1, day-3 and day-5 from T382L64 and T574L64 models. The contingency table illustrating the counts used in verification statistics for dichotomous forecasts and observations is stated in table 5. The verification is for rainfall excess of $0.5 \mathrm{~cm}$ in a day. Day-1 forecast of both the models are underpredicting over north-west India and overpredicting over Jammu and Kashmir regions. However, T382L64 shows substantial underprediction over north-west parts of India as compared with T574 in day- 1 and day- 3 forecasts. However, the feature is not apparently visible in day-5 forecasts. T574L64 shows higher skill over eastern and central regions in day-3 and day-5 forecasts.

To further examine the skill of both the models in predicting the rainfall intensities, bias scores are presented in figure 8 for rainfall excess of 3,4 and $5 \mathrm{~cm}$ in 24 hours. For rainfall thresholds starting from $3 \mathrm{~cm} /$ day, T574L64 shows higher skill than T382L64 in all day-1 to day-5 forecasts.

\subsection{Tropical cyclone relocation scheme}

Accurate prediction of tropical cyclones depends on the correct initial analysis of its position and strength. Data scarcity over oceanic region poses limitation on defining the initial position for tropical cyclone accurately. Assimilation of synthetic vortices in case of tropical cyclones were used operationally in NCMRWF since 1994, but the results were not always satisfactory. One of the reasons for this was the erroneous position, structure and intensity of the existing cyclone in the first guess. Often, cyclone in first guess is weak in strength and extends over large area compared to the observed cyclone, and further the model predicted centre is located away from the actual observed position. Thus, adding synthetic observations (cyclone bogus) generally leads to formation of two vortices in the same region, one from the first guess and the other from the synthetic data. The interaction between these two vortices
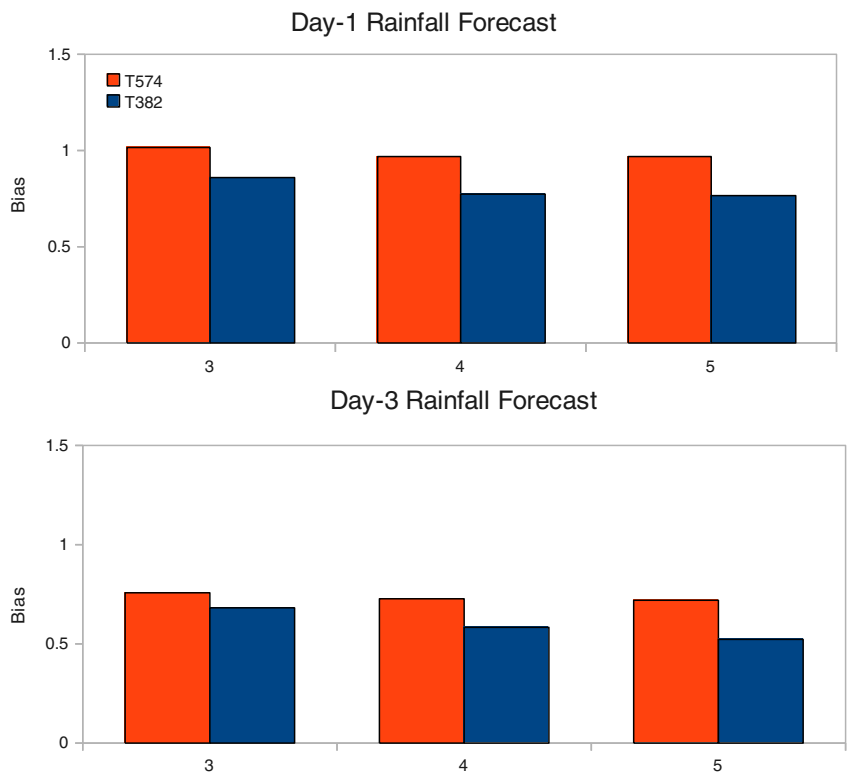

Day-5 Rainfall Forecast

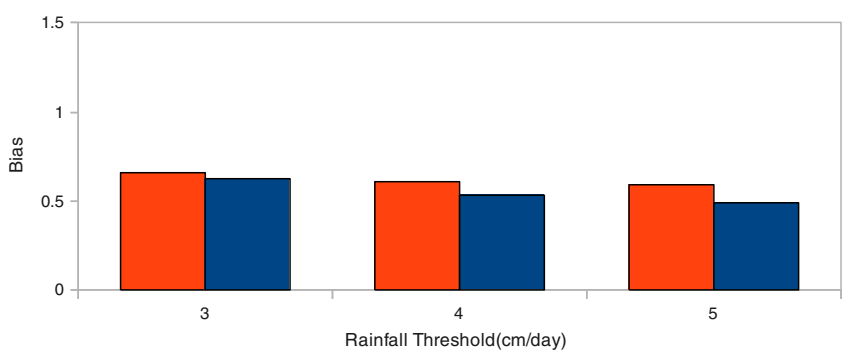

Figure 8. Frequency bias scores of the rainfall forecasts for different thresholds for both T382L64 (in red) and T574L64 (in blue) models for the period June-September 2011.

often leads to subsequent erroneous prediction. The tropical cyclone relocation scheme (Liu et al. 2000) was implemented at NCMRWF in T254L64 system during 2008. This scheme separates cyclone vortex from environmental fields in the first guesses (3-hr, 6-hr and 9-hr forecasts) used for subsequent analysis step, relocates the same to the observed cyclone position according to satellite estimated position reported in Joint Typhoon Warning Centre (JTWC) bulletins. If the predicted cyclone strength in the first guess fields is weaker than that of observed one, then the scheme also adds synthetic wind observations in the vicinity of the cyclone. Figure 9 depicts the track prediction errors of the tropical cyclone over Indian seas in T254L64, T382L64 and T574L64 modelling system during 2007 to 2011. It is clearly seen that the errors in the location of the tropical cyclones in the analysis (initial conditions) have reduced significantly after October 2008 (after the use of tropical cyclone relocation procedure in T254L64). Track errors are further reduced with increase in model resolution to T382 and T574. 


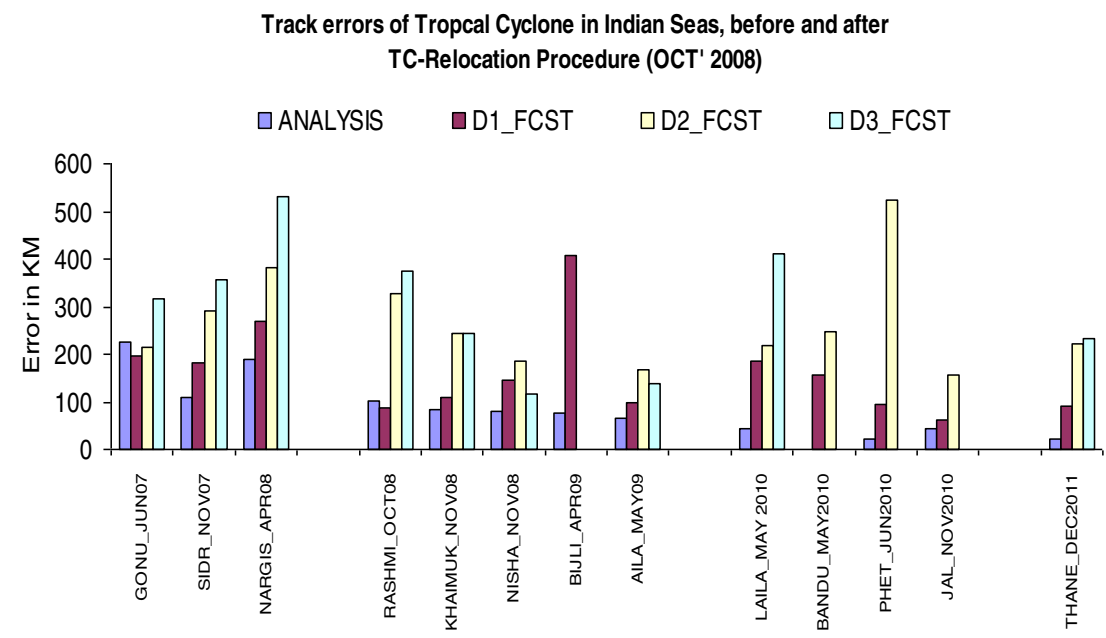

Figure 9. Track errors of tropical cyclones in Indian seas, before and after the implementation of the tropical cyclone relocation procedure in October 2008.

\section{Summary}

The successful implementation of the T574L64 GFS at NCMRWF makes India to be at par with major operational NWP centres. The new GFS represents a significant jump, not only in an improvement in the model physics, but also in the capability of decoding and assimilating more conventional and satellite observations. The comprehensive evaluation of the new system has shown improvements in many metrics. One of the major improvements is in the gain of 1 day in the skill of the forecasts as compared to T382L64. The tropical cyclone track errors have also reduced with increase in model resolution. India is also augmenting its current observational network and also planning to deploy various new observing systems. The upcoming INSAT-3D satellite with atmospheric sounder along with Megha-Tropiques satellite is going to provide a unique opportunity to assimilate direct satellite radiance data from an Indian satellite for improving the initial conditions and the forecasts subsequently.

\section{Acknowledgements}

The authors gratefully acknowledge National Center for Environmental Prediction (NCEP), USA for sharing the GFS/GSI codes. They also acknowledge Dr L Harenduprakash for his initial encouragement.

\section{References}

Alpert J C, Kanamitsu M, Caplan P M, Sela J G, White G H and Kalnay E 1988 Mountain induced gravity wave drag parameterization in the NMC medium-range forecast model; Proc. 8th Conf. NWP, Baltimore, MD.
Alpert J C, Hong S-Y and Kim Y-J 1996 Sensitivity of cyclogenesis to lower troposphere enhancement of gravity wave drag using the Environmental Modeling Center medium range model; Proc. 11th Conf. NWP, Norfolk, pp. 322-323.

Asselin R 1972 Frequency filter for time integrations; Mon. Weather Rev. 100 487-490.

Charnock H 1955 Wind stress on a water surface; Quart. J. Roy. Meteor. Soc. 81 639-640.

Courtier P and Coauthors 1998 The ECMWF implementation of the three-dimensional variational assimilation (3D-Var). I: Formulation; Quart. J. Roy. Meteorol. Soc. 124 1783-1807.

Ek M B, Mitchell K E, Lin Y, Rogers E, Grunmann P, Koren V, Gayno G and Tarplay J D 2003 Implementation of the Noah land-use model advances in the NCEP operational mesoscale Eta model; J. Geophys. Res. 108 8851, doi: 10.1029/2002JD003296.

Environmental Modeling Centre 2003 The GFS Atmospheric Model; NCEP Office Note 44212.

Han J and Pan H-L 2006 Sensitivity of hurricane intensity forecast to convective momentum transport parameterisation; Mon. Weather Rev. 134 664-674.

Han J and Pan H-L 2010 Revision of convection and vertical diffusion schemes in the NCEP global forecast system; NCEP Office Note $\mathbf{4 6 4}$ 42, http://www.emc.ncep.noaa. gov/officenotes/newernotes/on464.pdf.

Hong S-Y and Pan H-L 1996 Nonlocal boundary layer vertical diffusion in a medium-range forecast model; Mon. Weather Rev. 124 2322-2339.

Hou Y-T, Campana K-A and Yang S-K 1996 Shortwave radiation calculations in the NCEP's global model. International Radiation Symposium, IRS-96, August 19-24, Fairbanks, AL.

Kalnay E, Kanamitsu M and Baker W E 1990 Global numerical weather prediction at the National Meteorological Centre; Bull. Am. Meteor. Soc. 71 1410-1428.

Kalnay E, Lord S J and McPherson R D 1998 Maturity of operational numerical weather prediction: Medium range; Bull. Am. Meteor. Soc. 79 2753-2892.

Kanamitsu M 1989 Description of the NMC global data assimilation and forecast system; Wea. Forecasting 4 335-342.

Kanamitsu M, Alpert J C, Campana K A, Caplan P M, Deaven D G, Iredell M, Katz B, Pan H L, Sela J and White G H 1991 Recent changes implemented into 
the global forecast system at NMC; Wea. Forecasting 6 425-435.

Kessler E 1969 On the distribution and continuity of water substance in atmospheric circulation; Meteor. Monographs 1084.

Kim Y-J and Arakawa A 1995 Improvement of orographic gravity wave parameterization using a mesoscale gravity wave model; J. Atmos. Sci. 52 1875-1902.

Liu Q, Marchok T, Pan H, Bender M and Lord S 2000 Improvements in hurricane initialization and forecasting at NCEP with global and regional (GFDL) models; NCEP/EMC Tech. Procedures Bull. 472 7, http: //205.156.54.206/om/tpb/472.htm.

Lock A P, Brown A R, Bush M R, Martin G M and Smith R N B 2000 A new boundary layer mixing scheme. Part-I: Scheme description and single column model tests; Mon. Weather Rev. 128 3187-3199.

Lott F and Miller M J 1997 A new subgrid-scale orographic drag parameterization: Its performance and testing; Quart. J. Roy Meteor. Soc. 123 101-127.

Mlawer E J, Taubman S J, Brown P D, Iacono M J and Clough S A 1997 Radiative transfer for inhomogeneous atmospheres: RRTM, a validated correlated-K model for the longwave; J. Geophys. Res. 102(D14) 16,663-16,682.

Mlawer E J, Clough S A, Brown P D and Tobin D C 1998 Collision-induced effects and the water vapor continuum; In: Proceedings of the Eighth Atmospheric Radiation Measurement (ARM) Science Team Meeting, DOE/ER-0738, US Department of Energy, Washington DC, pp. 503-511.

Moorthi S, Pan H L and Caplan P 2001 Changes to the 2001 NCEP operational MRF/AVN global analysis/ forecast system; NWS Technical Procedures Bulletin $\mathbf{4 8 4}$ 14, http://www.nws.noaa.gov/om/tpb/484.htm.

Pan H-L and Wu W-S 1995 Implementing a Mass Flux Convection Parameterization Package for the NMC MediumRange Forecast Model; NMC Office Note 40940.

Parrish D F and Derber J C 1992 The National Meteorological Center's Spectral Statistical Interpolation Analysis System; Mon. Weather Rev. 120 1747-1763.

Prasad V S, Saji Mohandas, Munmun Das Gupta, Rajagopal E N and Surya Kanti Datta 2011 Implementation of upgraded Global Forecasting Systems (T382L64 and T574L64) at NCMRWF, NCMRWF Technical Report No. NCMR/TR/5/2011 May 2011, 72p, http://www.ncmrwf.gov.in/ncmrwf/gfs_report_final.pdf.

Rajagopal E N, Das Gupta M, Saji Mohandas Prasad V S, John P George, Iyengar G R and Preeven Kumar D 2007 Implementation of T254L64 Global Forecast System at NCMRWF; NMRF/TR/1/2007, 42p, http:// www.ncmrwf.gov.in/ncmrwf/gfs_report_T254.pdf.
Saha Suranjana et al. 2010 The NCEP climate forecast system reanalysis; Bull. Am. Meteor. Soc. 91 1015-1057, doi: 10.1175/2010BAMS3001.1.

Sato Hansen J E, McCormick M P and Pollack J B 1993 Stratospheric aerosol optocal depth 1850-1990; J. Geophys. Res. 98 22,987-22,994.

Sela J 2009 Implementation of the sigma pressure hybrid coordinate into GFS; NCEP Office Note \# 461 [available at http://www.emc.ncep.noaa.gov/officenotes/FullTOC. html\#2000].

Sundqvist H, Berge E and Kristjansson J E 1989 Condensation and cloud studies with mesoscale numerical weather prediction model; Mon. Weather Rev. 117 1641-1757.

Surya Kanti Dutta and Prasad V S 2011 Impact of gridpoint statistical interpolation scheme over Indian region; J. Earth Syst. Sci. 120(6) 1095-1112.

Tiedtke M 1983 The sensitivity of the time-mean largescale flow to cumulus convection in the ECMWF model; In: Proceedings of the ECMWF Workshop on Convection in Large-Scale Models, 28 November-1 December 1983, European Centre for Medium-Range Weather Forecasts, Reading, England, pp. 297-316.

Winton M 2000 A reformulated three-layer sea ice model; J. Atmos. Oceanic Technol. 17 525-531.

WMO-No. 485 Manual on the Global Data-processing and Forecasting System.

Wu Wan-Shu, James Purser R and David F Parrish 2002 Three-dimensional variational analysis with spatially inhomogeneous covariances; Mon. Weather Rev. 130 2905-2916.

Xu K M and Randall D A 1996 A semiempirical cloudiness parameterization for use in climate models; J. Atmos. Sci. 53 3084-3102.

Yang F, Mitchell K, Hou Y-T, Dai Y, Deng X, Wang Z and Liang X-Z 2008 Dependence of land surface albedo on solar zenith angle: Observations and model parameterisations; J. Climate Appl. Meteor. 47 2963-2982.

Yang F et al. 2009 On the negative water vapour in the NCEP GFS: Sources and solutions; 23rd Conference in Weather Analysis and Forecasting/19th Conference on Numerical Weather Prediction, Am. Meteor. Soc. 1-5 June 2009, Omaha, NE.

Zeng X, Zhao M and Dickinson R E 1998 Intercomparison of bulk aerodynamical algorithms for the computation of sea surface fluxes using TOGA COARE and TAO data; J. Climate $112628-2644$.

Zhao Q Y and Carr F H 1997 A prognostic cloud scheme for operational NWP models; Mon. Weather Rev. 125 1931-1953. 